\title{
765.
}

\section{ON THE FLEXURE AND EQUILIBRIUM OF A SKEW SURFACE.}

[From the Proceedings of the London Mathematical Society, vol. xII. (1881), pp. 103-108. Read March 10, 1881.]

THE skew surface is taken to be such that the strip between two consecutive generating lines is rigid, and that the flexure takes place by the rotation of the strips about the generating lines successively. The theory of the flexure is well known, but I am not aware that the theory of the equilibrium of such a surface, when acted upon by any given forces, has been considered; it is, however, a question which presents itself naturally in connexion with those relating to other continuous bodies treated of in the Mécanique Analytique, and forms a good example of the principles made use of.

To begin with the mechanical theory: we may regard the forces as acting on the generating lines regarded as material lines; and if for an element of mass $d m$, coordinates $(x, y, z)$ of a particular generating line $G$, the forces parallel to the axes are $X^{\prime}, Y^{\prime}, Z^{\prime}$, then the corresponding term in the equation of equilibrium is

$$
S\left(X^{\prime} \delta x+Y^{\prime} \delta y+Z^{\prime} \delta z\right) d m
$$

and observing that there are (as will afterwards appear) five geometrical conditions, which I represent by $U_{1}=0, U_{2}=0, \ldots, U_{5}=0$, the equation of equilibrium is

$$
S\left\{\left(X^{\prime} \delta x+Y^{\prime} \delta y+Z^{\prime} \delta z\right) d m+T_{1} \delta U_{1}+T_{2} \delta U_{2}+T_{3} \delta U_{3}+T_{4} \delta U_{4}+T_{5} \delta U_{5}\right\}=0
$$

where $T_{1}, T_{2}, \ldots, T_{5}$ are the indeterminate multipliers, representing colligation-forces which correspond to the five geometrical conditions respectively.

Taking $(\xi, \eta, \zeta)$ for the coordinates of a particular point $P$ on the generating line; $p, q, r$ for the cos-inclinations of the line (whence $U_{1}=p^{2}+q^{2}+r^{2}-1=0$ is one of the geometrical relations), and $\rho$ for the distance of $d m$ from $P$, we have

$$
\begin{aligned}
x, \quad y, \quad z & =\xi+\rho p, \quad \eta+\rho q, \quad \zeta+\rho r, \\
\delta x, \delta y, \delta z & =\delta \xi+\rho \delta p, \delta \eta+\rho \delta q, \delta \zeta+\rho \delta r .
\end{aligned}
$$


The summation $S$ extends first to the different points of the generating line, and then to the different generating lines; applying it first to the particular generating line, we write

$$
S X^{\prime} d m, \quad S Y^{\prime} d m, \quad S Z^{\prime} d m, \quad S X^{\prime} \rho d m, \quad S Y^{\prime} \rho d m, \quad S Z^{\prime} \rho d m
$$

$=\quad X, \quad Y, \quad Z, \quad L, \quad M, \quad N$,

where $X, Y, Z$ are the whole forces, and $L, M, N$ the whole moments about the point $P$, for the generating line $G$; retaining the same summatory symbol $S$, as now referring to the different generating lines, the equation becomes

$$
S\left\{X \delta \xi+Y \delta \eta+Z \delta \zeta+L \delta p+M \delta q+N \delta r+T_{1} \delta U_{1}+\ldots+T_{5} \delta U_{5}\right\}=0 .
$$

We have now to consider the geometrical theory of the flexure. Taking on the skew surface an arbitrary curve cutting each generating line $G$ in a point $P$, coordinates $(\xi, \eta, \zeta)$, and taking $\sigma$ for the distance along the curve of the point $P$ from a fixed point of the curve; also $p, q, r$, as before, for the cos-inclinations of the generating line $G$, then when the surface is in a determinate state, $\xi, \eta, \zeta, p, q, r$ are given functions of $\sigma$; but these functions vary with the flexure of the surface, with, however, certain relations unaffected by the flexure; and the problem is to find first these relations. As already mentioned, one of them is $p^{2}+q^{2}+r^{2}-1=0$.

Taking $P^{\prime}$ as the consecutive point on the curve, so that the direction of the element $P P^{\prime}$ is that of the tangent $P T$ at $P$, it is convenient to write $l, m, n$ for the cosine-inclinations of the tangent; we have, it is clear,

$$
l, m, n=\frac{d \xi}{d \sigma}, \frac{d \eta}{d \sigma}, \frac{d \zeta}{d \sigma} ; l^{2}+m^{2}+n^{2}-1=0 .
$$

The conditions in order to the rigidity of the strip, are that the angles GPP', $G^{\prime} P^{\prime} P\left(=180^{\circ}-G^{\prime} P^{\prime} T\right)$, and the inclination $G^{\prime} P^{\prime}$ to $G P$, shall have given values,

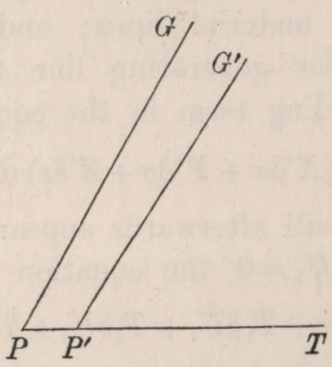

variable it may be from strip to strip-that is, these values must be given functions of $\sigma$. Taking $\angle G P T=I$, the value of $G^{\prime} P^{\prime} T$ can differ only infinitesimally from that of GPT, and we take it to be $G^{\prime} P^{\prime} T=I-\Omega d \sigma$; also the inclination $G P$ to $G^{\prime} P^{\prime}$ is an infinitesimal, $=\Theta d \sigma$ : we have $I, \Omega, \Theta$ given functions of $\sigma$. It is to be remarked that these conditions imply, inclination of $G^{\prime} P^{\prime}$ to tangent plane GPT at $P$ has a given value $\Lambda d \sigma$; in fact, if through $P$ we draw a line $P_{\gamma}$ parallel to $P^{\prime} G^{\prime}$, then, if $P$ is regarded as the centre of a sphere which meets $P G, P \gamma, P T$ in the 
points $g, g^{\prime}, t$ respectively, we have a spherical triangle $g g^{\prime} t$, the sides of which are $I-\Omega d \sigma, I$, and $\Theta d \sigma$, and of which the perpendicular $g^{\prime} m$ is $=\Lambda d \sigma$; we have thus an infinitesimal right-angled triangle, the base and altitude of which are $\Omega d \sigma, \Lambda d \sigma$,

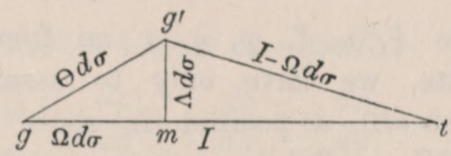

and the hypothenuse is $\Theta d \sigma$; whence $\Theta^{2}=\Omega^{2}+\Lambda^{2}$. In the case of the developable surface $\Lambda=0$ and $\Theta=\Omega$. It may be remarked that, when the curve on the skew surface is the line of striction, we have $\Omega=0$; in fact, taking $P$ to be on the line of striction, the line

$$
\frac{X-\xi}{q r^{\prime}-q^{\prime} r}=\frac{Y-\eta}{r p^{\prime}-r^{\prime} p}=\frac{Z-\zeta}{p q^{\prime}-p^{\prime} q},
$$

through $(\xi, \eta, \zeta)$ at right angles to the two generating lines, meets the consecutive generating line $X, Y, Z=\xi^{\prime}+\rho p^{\prime}, \eta^{\prime}+\rho q^{\prime}, \zeta^{\prime}+\rho r^{\prime}$; and the condition that this may be so is easily found to be $\Omega=0$.

Take, for a moment, $p^{\prime}, q^{\prime}, r^{\prime}$ for the cos-inclinations of the consecutive generating line $P^{\prime} G^{\prime}$; we have

$$
\begin{aligned}
& l p+m q+n r=\cos I, \\
& l p^{\prime}+m q^{\prime}+n r^{\prime}=\cos (I-\Omega d \sigma), \\
& p p^{\prime}+q q^{\prime}+r r^{\prime}=\cos \Theta d \sigma ;
\end{aligned}
$$

and then writing $p^{\prime}, q^{\prime}, r^{\prime}=p+d p, q+d q, r+d r$, and observing that the equation $p^{\prime 2}+q^{\prime 2}+r^{\prime 2}=1$ gives

$$
p d p+q d q+r d r=-\frac{1}{2}\left(d p^{2}+d q^{2}+d r^{2}\right)
$$

these equations and the before-mentioned two equations become

$$
\begin{aligned}
& \left(U_{1}\right) \quad p^{2}+q^{2}+r^{2}-1=0, \\
& \left(U_{2}\right) \quad l^{2}+m^{2}+n^{2}-1=0, \\
& \left(U_{3}\right) \quad l p+m q+n r-\cos I=0, \\
& \left(U_{4}\right) \quad l d p+m d q+n d r-\Omega \sin I d \sigma=0, \\
& \left(U_{5}\right) d p^{2}+d q^{2}+d r^{2}-\Theta^{2} d \sigma^{2}=0,
\end{aligned}
$$

which equations, considering therein $l, m, n$ as standing for their values $\frac{d \xi}{d \sigma}, \frac{d \eta}{d \sigma}, \frac{d \zeta}{d \sigma}$, are the geometrical relations which connect the six variables $\xi, \eta, \zeta, p, q, r$, considered as functions of $\sigma$. And in these equations $I, \Omega, \Theta$ denote given functions of $\sigma$, invariable by any flexure of the surface.

To complete the geometrical theory, it is to be observed that we can by flexure bring the generating lines of the surface to be parallel to those of any given cone 
$C(p, q, r)=0$, where $C(p, q, r)$ denotes a homogeneous function of $(p, q, r)$. Hence, joining to the foregoing five equations this new equation

$$
C(p, q, r)=0,
$$

these six equations determine $\xi, \eta, \zeta, p, q, r$ as functions of $\sigma$. To make the solution completely determinate, we have only to assume for the point $P$, which corresponds, say, to the value $\sigma=0$, a position in space at pleasure, and to take the corresponding generating line $P G$ parallel to a generating line, at pleasure, of the cone.

As an example, writing $\gamma$ to denote an arbitrary constant angle, if the invariable conditions are

$$
I=\gamma, \quad \Theta=\sin \gamma, \quad \Omega=0,
$$

then the five equations are

$$
\begin{array}{rlr}
p^{2}+q^{2}+r^{2}-1 & =0 \\
l^{2}+m^{2}+n^{2}-1 & =0 \\
l p+m q+n r-\cos \gamma & =0 \\
d p^{2}+d q^{2}+d r^{2}-\sin ^{2} \gamma d \sigma^{2} & =0 \\
l d p+m d q+n d r & =0
\end{array}
$$

We assume first

$$
C(p, q, r)=p^{2}+q^{2}-r^{2} \tan ^{2} \gamma,=0
$$

and secondly

$$
C(p, q, r)=r,=0 .
$$

Then, in the former case, we find the solution

$p, q, r=-\sin \gamma \sin \sigma, \sin \gamma \cos \sigma, \cos \gamma ;$

$$
\xi, \eta, \zeta=\cos \sigma, \sin \sigma, 0 \text {; }
$$

giving

$$
x, y, z=\cos \sigma-\rho \sin \gamma \sin \sigma, \sin \sigma+\rho \sin \gamma \cos \sigma, \cos \gamma
$$

and consequently

$$
x^{2}+y^{2}-z^{2} \tan ^{2} \gamma=0,
$$

the hyperboloid of revolution. And, in the latter case,

$$
\begin{aligned}
& p, q, r=\cos (\sigma \sin \gamma), \sin (\sigma \sin \gamma), 0, \\
& \xi, \eta, \zeta=\cot \gamma \sin (\sigma \sin \gamma),-\cot \gamma \cos (\sigma \sin \gamma), \sigma \sin \gamma,
\end{aligned}
$$

that is,

whence

$$
x, y=\cot \gamma \sin z+\rho \cos z,-\cot \gamma \cos z+\rho \sin z,
$$

$$
x \sin z-y \cos z=\cot \gamma,
$$

a skew helicoid generated by horizontal tangents of the cylinder $x^{2}+y^{2}=\cot ^{2} \gamma$. This is a known deformation of the hyperboloid. 
Returning now to the mechanical problem, we have to consider the terms

$$
\begin{aligned}
S & . T_{1} \delta \frac{1}{2}\left(p^{2}+q^{2}+r^{2}-1\right) \\
& +T_{2} \delta \frac{1}{2}\left(l^{2}+m^{2}+n^{2}-1\right) \\
& +T_{3} \delta(l p+m q+n r-\cos I) \\
& +T_{4} \delta\left(l \frac{d p}{d \sigma}+m \frac{d q}{d \sigma}+n \frac{d r}{d \sigma} \Omega \sin I\right) \\
& +T_{5} \delta \frac{1}{4}\left\{\left(\frac{d p}{d \sigma}\right)^{2}+\left(\frac{d q}{d \sigma}\right)^{2}+\left(\frac{d r}{d \sigma}\right)^{2}-\Theta^{2}\right\} .
\end{aligned}
$$

The first term gives, under the sign $S$,

$$
T_{1}(p \delta p+q \delta q+r \delta r) .
$$

The second term gives, in the first instance,

or, since in general

$$
\frac{T_{2}}{d \sigma}(l d \delta \xi+m d \delta \eta+n d \delta \zeta)
$$

$$
S \Omega d \delta \xi=\Omega^{\prime \prime} \delta \xi^{\prime \prime}-\Omega^{\prime} \delta \xi^{\prime}+S(-d \Omega . \delta \xi),
$$

then, attending only to the terms under the sign $S$, these are

$$
=-\frac{d}{d \sigma} T_{2} l . \delta \xi-\frac{d}{d \sigma} T_{2} m . \delta \eta-\frac{d}{d \sigma} T_{2} n . \delta \zeta .
$$

The third term gives

where the second line,

$$
\begin{array}{r}
T_{3}(l \delta p+m \delta q+n \delta r) \\
+T_{3}(p \delta l+q \delta m+r \delta n),
\end{array}
$$

$$
=\frac{T_{3}}{d \sigma}(p d \delta \xi+q d \delta \eta+r d \delta \zeta),
$$

attending only to the terms under the sign $S$, gives

The fourth term gives

$$
-\frac{d}{d \sigma} T_{3} p \cdot \delta \xi-\frac{d}{d \sigma} T_{3} q \cdot \delta \eta-\frac{d}{d \sigma} T_{3} r . \delta \zeta .
$$

$$
\begin{array}{r}
T_{4}\left(\frac{d p}{d \sigma} \delta l+\frac{d q}{d \sigma} \delta m+\frac{d r}{d \sigma} \delta n\right) \\
+T_{4}\left(\frac{l}{d \sigma} d \delta p+\frac{m}{d \sigma} d \delta q+\frac{n}{d \sigma} d \delta r\right)
\end{array}
$$

where the first line, written under the form

$$
\frac{T_{4}}{d \sigma}\left(\frac{d p}{d \sigma} d \delta \xi+\frac{d q}{d \sigma} d \delta \eta+\frac{d r}{d \sigma} d \delta \zeta\right),
$$

and attending only to the terms under the sign $S$, gives

$$
-\frac{d}{d \sigma}\left(T_{4} \frac{d p}{d \sigma}\right) \cdot \delta \xi-\frac{d}{d \sigma}\left(T_{4} \frac{d q}{d \sigma}\right) \cdot \delta \eta-\frac{d}{d \sigma}\left(T_{4} \frac{d r}{d \sigma}\right) \cdot \delta \zeta ;
$$

C. XI. 
and the second line, attending in like manner only to the terms under the sign $S$, gives

$$
-\frac{d}{d \sigma} T_{4} l . \delta p-\frac{d}{d \sigma} T_{4} m . \delta q-\frac{d}{d \sigma} T_{4} n . \delta r .
$$

The fifth term, written under the form

$$
\frac{T_{5}}{d \sigma}\left(\frac{d p}{d \sigma} d \delta p+\frac{d q}{d \sigma} d \delta q+\frac{d r}{d \sigma} d \delta r\right)
$$

and attending only to the terms under the sign $S$, gives

$$
-\frac{d}{d \sigma} T_{5} \frac{d p}{d \sigma} \cdot \delta p-\frac{d}{d \sigma} T_{5} \frac{d q}{d \sigma} \cdot \delta q-\frac{d}{d \sigma} T_{5} \frac{d r}{d \sigma} \cdot \delta r ;
$$

where in each case I have marked with an asterisk the lines which present themselves in the final result.

Hence, joining to the foregoing the force-terms

$$
X \delta \xi+Y \delta \eta+Z \delta \zeta+L \delta p+M \delta q+N \delta r
$$

and equating to zero the coefficients of $\delta \xi, \delta \eta, \delta \zeta, \delta p, \delta q, \delta r$ respectively, we have

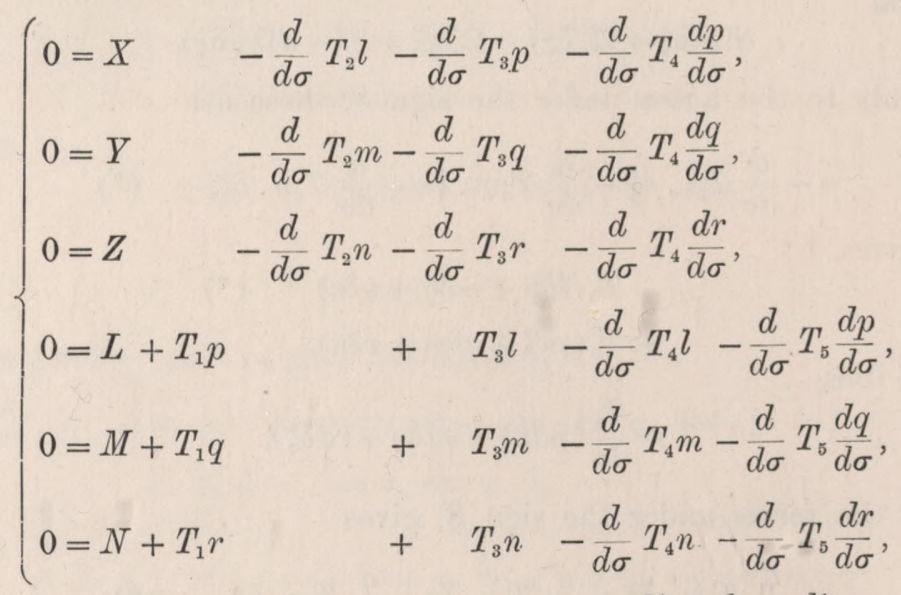

where it will be recollected that $l, m, n$ stand for $\frac{d \xi}{d \sigma}, \frac{d \eta}{d \sigma}, \frac{d \zeta}{d \sigma}$, the variables being $\xi, \eta, \zeta, p, q, r$, and $\sigma$. The elimination of $T_{1}, T_{2}, \ldots, T_{5}$ from the six equations should lead to a relation between $\xi, \eta, \zeta, p, q, r$, which, with the foregoing five relations, would determine the six variables $\xi, \eta, \zeta, p, q, r$ in terms of $\sigma$.

In particular, the forces and moments $X, Y, Z, L, M, N$ may all of them vanish; assuming that $T_{1}, T_{2}, \ldots, T_{5}$ do not all of them vanish, we still have the sixth relation, which (with the foregoing five relations) determines $\xi, \eta, \zeta, p, q, r$ in terms of $\sigma$; and it is to be remarked that the problem in question, of the figure of equilibrium of the skew surface not acted upon by any forces, is analogous to that of the geodesic line in space; only whilst here the solution is, curve a straight line, the solution for the case of the skew surface depends upon equations of a complex enough form; in the case of the developable surface, the required figure is of course the plane. 